\title{
Clinical Assessment of Dysphagia in Neurodegeneration (CADN): Development, validity and reliability of a bedside tool for dysphagia assessment
}

Adam P. Vogel ${ }^{1,2}(\mathrm{PhD})^{*}$, Natalie Rommel ${ }^{1,3}$ (MSc), Carina Sauer ${ }^{3}(\mathrm{BSc})$, Marius Horger (MD) ${ }^{4}$, Patrick Krumm (MD) ${ }^{4}$, Marc Himmelbach (PhD) ${ }^{6-7}$, Matthis Synofzik ${ }^{1,5}$ (MD)

${ }^{1}$ Department of Neurodegeneration, Hertie Institute for Clinical Brain Research \& Center for Neurology, University Hospital Tübingen, Germany

${ }^{2}$ Centre for Neuroscience of Speech, The University of Melbourne, Victoria, Australia

${ }^{3}$ TherapieZentrum, University Hospital Tübingen, Germany

${ }^{4}$ Department of Diagnostic and Interventional Radiology, University Hospital Tübingen, Germany

${ }^{5}$ Center for Neurodegenerative Diseases (DZNE), University of Tübingen, Germany

${ }^{6}$ Section of Neuropsychology, Department of Cognitive Neurology, Hertie Institute for

Clinical Brain Research \& Center for Neurology, University Hospital Tübingen, Germany

${ }^{7}$ Centre for Integrative Neuroscience, University of Tübingen, Germany

Manuscript word count: 4633 (including headings)

Abstract word count: 261

Word count for title: 18

Number of references: 52

Number of figures: 1

Number of tables: 3

Supplementary materials: 2 documents

Corresponding author:

A/Prof Adam Vogel

Centre for Neuroscience of Speech

The University of Melbourne

550 Swanston Street, Parkville

Melbourne VIC 3010

Australia

Phone: +61 390355334

Email:vogela@unimelb.edu.au

Keywords: swallowing, clinical bedside assessment, Parkinson's disease, spinocerebellar ataxia, POLG, ARSACS, deglutition, clinical trials

Financial Disclosure/Conflict of Interest: None. 


\section{Abstract}

Background: Screening assessments for dysphagia are essential in neurodegenerative disease. Yet there are no purpose-built tools to quantify swallowing deficits at bedside or in clinical trials. A quantifiable, brief, easy to administer assessment that measures the impact of dysphagia and predicts the presence or absence of aspiration is needed.

Methods: The Clinical Assessment of Dysphagia in Neurodegeneration (CADN) was designed by a multidisciplinary team (neurology, neuropsychology, speech pathology) validated against strict methodological criteria in two neurodegenerative diseases, Parkinson's disease (PD) and degenerative ataxia (DA). CADN comprises two parts, an anamnesis (part one) and consumption (part two). Two thirds of patients were assessed using reference tests, the SWAL-QOL symptoms subscale (part one) and videofluoroscopic assessment of swallowing (part two). CADN has 11 items and can be administered and scored in an average of 7 minutes. Test-retest reliability was established using correlation and Bland-Altman plots.

Results: 125 patients with a neurodegenerative disease were recruited; 60 PD and 65 DA. Validity was established using ROC graphs and correlations. CADN has sensitivity of 79\% and $84 \%$ and specificity $71 \%$ and $69 \%$ for parts one and two respectively. Significant correlations with disease severity were also observed $(p<.001)$ for PD with small to large associations between disease severity and CADN scores for DA. Cut off scores were identified that signal the presence of clinically meaningful dysphagia symptomatology and risk of aspiration.

Conclusions: The CADN is a reliable, valid, brief, quantifiable, and easily deployed assessment of swallowing in neurodegenerative disease. It is thus ideally suited for both clinical bedside assessment and future multicentre clinical trials in neurodegenerative disease. 


\section{Introduction}

Swallowing deficits (dysphagia) following the onset of progressive neurodegenerative disease (NDD) are common [1,2]. Difficulties eating and drinking can have pervasive and devastating effects on the health and quality of life of the individual [3]. Dysphagia can lead to aspiration pneumonia and subsequent death in a number of NDD groups including Parkinson's disease [4], Huntington's disease [5, 6], amyotrophic lateral sclerosis [7] and hereditary ataxias $[1,8-10]$ making identification and measurement of dysphagia a key clinical and research priority in neurodegeneration.

Bedside assessments of dysphagia in clinical settings are often unstandardized, relying on subjective judgments of the clinician rather than quantitative protocols. Clinical centres regularly develop their own informal screening tools with unknown psychometric properties [11]. These instruments are not suitable for use in clinical research, and may not be appropriate for day to day clinical practices given the lack of information on their reliability, sensitivity and specificity to impairment. Dysphagia assessments in experimental settings (e.g., drug trials for progressive neurological disorders) are either absent, or are focussed on the presence or absence of aspiration only. Where validated and standardised assessments are available [12-20], they present with mixed sensitivity, specificity and utility (described here as face validity, ease of use, timeliness, cost, and multiple language options).

Two reviews of clinical bedside assessments conducted in the past decade examined the quality of 49 tools designed to screen swallowing impairment in neurological populations $[21,22]$. Only nine of those tests met predetermined criteria relating to adequate validity, generalizability and reliability (developed by the Dutch Cochrane Centre (http://www.cochrane.nl) as well as sensitivity and specificity $\geq 50 \%$. Each tool aimed to determine the presence or absence of aspiration through trialling water and/or a variety of 
consistencies and viscosities, some including simple oral motor tasks such as lateral tongue movement and others noting signs of suspected aspiration including coughing, choking or gagging or including pulse oximetry $[12,13,15,16,20,23-26]$. Six of those assessments were validated exclusively in stroke populations, with three including NDD in addition to stroke patients $[12,15,16]$. Of those tools that considered NDD patients, three examined swallowing through consumption only [Clavè et al. [12] trialled different viscosities and volumes delivered via syringe and simultaneous pulse oximetry; Wakasugi et al. [16] aimed to determine the presence of silent aspiration through a cough test where patients inhaled mist of citric acid-physiologic saline orally for $1 \mathrm{~min}$ with an ultrasonic nebulizer; Suiter and Leder [15] required all participants to drink $90 \mathrm{cc}$ of water without interruption], with the fourth by Logemann et al [23], the 28 item Northwestern Dysphagia Patient Check Sheet (NDPC), considering medical history (e.g., history of recurrent pneumonia), behavioural variables (e.g., alertness), gross motor function (e.g., posture), oral motor function and observations during consumption of different viscosities, all within a two category response (safe/not safe). Considering all tools with strong psychometric properties together, the NDPC offers the most comprehensive assessment of dysphagia within the context of a bedside screening. Yet the NDPC does not provide scope for quantifying the severity of deficits, frequency of swallowing deficits nor elicit responses relating to compensatory eating and drinking behaviours such as modified diet. Its length (28 items plus consumption tasks) also restricts its utility as a quick bedside tool for evaluating dysphagia in clinical routine and multicenter trials in NDD.

The remaining clinical bedside assessments did not consider or quantify secondary behaviours relating to swallowing including modified diet, presence of chest infections, or level of independence during mealtimes. Data on these behaviours are essential for 
determining how the swallowing deficits are managed. They also provide information on how deficits impact the patient beyond the impairment itself.

The aim of the current study was to develop and validate a clinical assessment of dysphagia for use in NDD populations. Existing options available to clinicians do not provide adequate means for quantifying the nature and severity of dysphagia in these progressive, usually multisystemic disorders. Here we seek to provide evidence for a valid, reliable and sensitive tool for evaluating dysphagia which builds on the strengths of existing swallowing screeners designed to identify suspected aspiration/penetration while incorporating quantitative data on the impact of feeding related activities. Development of an assessment that describes the impact of dysphagic symptoms by allocating a cumulative severity value to the patient's swallow as well as characterises the nature of the deficit and how dysphagia manifests in the patient fills an important gap in the assessment battery of clinicians and researchers working in NDDs.

\section{Methods}

Tool Development

The Clinical Assessment of Dysphagia in Neurodegeneration (CADN) was developed following a thorough literature search and related systematic review of dysphagia self-report questionnaires conducted by us [27], which revealed an absence of clinical screening assessments of dysphagia suitable for progressive neurological disorders. We focused on building a quick (CADN administration and scoring takes on average 7 minutes as determined by measuring administration time of three assessors to three participants each with an average of 7.4 minutes with a range 6-10 minutes) bedside tool (CADN has 11-items) to provide information on both aspiration risk but also feeding and compensatory behaviours. 
It was designed to serve a threefold purpose: 1. to quantify dysphagia as a clinical endpoint; 2. to inform clinical care through identification of feeding behaviours; 3 . to predict the presence or absence of aspiration signalling the need for further investigation via videofluoroscopic swallowing study (VFSS) or Fibreoptic endoscopic evaluation of swallowing (FEES).

The CADN was developed by a multidisciplinary team ( 3 speech-language pathologists, 1 neurologist, and 1 neuropsychologist) with clinical experience in progressive neurological disorders, in particular NDD. The first version of CADN included 13 items with the additional two items examining lip and tongue function in isolation (not during consumption). After piloting this extended version of the CADN within the clinic, the development group decided to remove the two 'oral motor' exam items to produce the 11 item version evaluated here. Oral motor items were considered outside of the remit of CADN and could be adequately addressed in other validated and widely used tools such as the Frenchay Dysarthria Assessment - 2 [28]. These items also added time to the assessment, reducing its utility. Severity scores for each of the 11 items of the CADN, ranging from 0 (no impairment) to 4 allow quantification of changes in function resulting from disease progression or treatment response. The CADN is split into two parts, a clinical case history (anamnesis) and a consumption part (see Supplement 1), each allowing quantification of different aspects of traditional swallowing assessments. Part one includes eight questions on frequency of coughing or choking on liquids and solids, saliva management, frequency of chest infections, assistance eating and drinking and diet modification (due to swallowing problems). Part two builds on existing aspiration risk tools by asking patients to drink water, and complete puree and biscuit trials. Water trials and different viscosities were included in the consumption part of the CADN as trial swallow tests with different viscosities have 
higher sensitivities and comparable specificities than water alone [21]. Further, different viscosities serve to inform clinicians' post assessment management. A discontinuation rule allows omission of the biscuit trial in patients with a severe impairment (score of 4) during the water or puree tasks.

A statistician based at the Hertie Institute for Clinical Brain Research, Tübingen, was consulted during development of the scoring matrix for CADN. The initial version of CADN used the highest score of each part to calculate the total score. However, that method led to implausible results, e.g. a patient who only coughs on water received the same score as a patient who coughs on all textures/consistencies. The final, validated version builds on the highest score by adding +1 for every 'moderate' or 'severe' score, ensuring CADN is sensitive to severe presentations. Moreover, the scores associated with descriptive terms were altered to reflect their meaning, i.e., patients with no impairment were able to receive scores of 0 . Scale categories that increase in line with increasing levels of severity allows for quantitative matching of clinical impressions with severity scores thus maximising the clinical interpretability of the tool. We also evaluated the instructions and scoring components of CADN with naive users, ensuring users unfamiliar with the tool were able to accurately score outcomes.

\section{Participants}

Assessors

Speech Language pathologists (SLPs) at the University Hospital Tübingen with more than two years' experience working with NDDs conducted the assessments. The assessors did not receive any additional training to conduct the assessment, but relied on the instructions contained in the tool. 


\section{Patients}

A consecutive series of patients with NDD admitted to the Department of Neurology, University Hospital Tübingen, Germany (both in-patients and out-patients) were recruited into the study from 2015 until 2016. Patients had to meet the diagnostic criteria of one of the following a neurodegenerative diseases out of one the following 2 groups: (1) Parkinson's disease (PD), or (2) degenerative ataxias (DA) (including autosomal-dominant spinocerebellar ataxias [SCA], Friedreich ataxia, Autosomal Recessive Spastic Ataxia of Charlevoix-Saguenay [ARSACS][29], mutations in the nuclear-encoded mitochondrial DNA polymerase gamma (POLG) related ataxias[30]), each diagnosed according to the respective consensus criteria (for PD) and to genetic confirmation (for SCAs, FRDA, ARSACS and POLG), respectively. All patients were included if they presented with some difficulty swallowing as determined by the consulting neurologist, and they were: $>18$ years old, able to provide informed consent, able to follow simple instructions, capable of sitting upright for anamnesis and examination for at least $20 \mathrm{~min}$ (in chair or wheelchair). Patients were excluded if they presented with a history of stroke, had surgery to the head or neck, received radiotherapy in the last 12 months, presented with dysphagia resulting from other known causes (e.g., somnolence), or had an allergy to radiopaque material (containing iodine). All patients were assessed for disease severity (PD: Hoehn and Yahr Scale (HY) [31]; group DA: Scale for the assessment and rating of ataxia [SARA][32]), Instrumental Activities of Daily Living [IADL][33] and, were screened for cognitive deficits using the Montreal Cognitive Assessment (MoCA)[34]. Patients receiving a score $>20$ were accepted into the study. Data from patients scoring $<20$ on the MoCA were also assessed using the CADN with the intent of determining its utility in individuals with cognitive impairment.

\section{Procedure}




\section{Reference tests and clinically meaningful endpoints}

Following informed consent, all patients were assessed using the CADN (index test). Twothirds of patients underwent a VFSS (reference test for part two of CADN) and completed a swallowing related quality of life survey (SWAL-QOL) [35] (reference test for part one of CADN). The VFSS was evaluated using the Penetration-Aspiration Scale (PAS) [36]. The PAS is a validated an 8-point, interval scale to describe penetration and aspiration events based on evaluation of VFSS. A clinically meaningful cut-off of $\geq 5$ was applied to these data. Scores equal to and above five suggest that material enters the airway, and either makes contact with or passes below the vocal folds during a swallow.

The SWAL-QOL is a validated self-report questionnaire. The Symptom scale of the SWALQOL includes 14 questions relating to coughing, choking, secretion management, etc. [35]. The score can be converted to a percentage where 100 equals no impairment. There are few validated self-report measures of swallowing function suitable for neurodegenerative populations [27]. The SWAL-QOL was considered the best equivalent of anamnesis available within the literature. It has been carefully validated [35, 37-39] and applied in a variety of neurodegenerative disorders including PD, Huntington's disease, Spinocerebellar Ataxia and Amyotrophic Lateral Sclerosis [40-44]. The SWAL-QOL was considered a suitable reference test on which to validate CADN part one based on several features (i.e., validity, reliability, coverage of relevant domains, interpretability, similarity to CADN part one, and wider acceptance and use by the scientific community and clinicians) as determined by a systematic review of self-reported swallowing assessments in progressive neurological disorders [27]. A conservative but meaningful cut-off, sensitive to any swallowing dysfunction, was determined by examining the scoring breakdown of the SWAL-QOL and by considering outcomes from control data of 112 healthy individuals without neurological illness or physical disability (mean age 44.64y, $\mathrm{SD}=17.14$; education average $14.61 \mathrm{y}, \mathrm{SD}=2.56$ ) [45]. 
The average of healthy controls based on our normative data for the symptoms score was 93 . Scores of $<89$ were considered indicative of dysfunction on the "Symptoms" scale of the SWAL-QOL. A score below 89 suggests that the participant has responded to all items (on average) that they 'Sometimes' or 'Hardly ever' (as opposed to 'Never') present with swallowing problems. This emphasis on sensitivity of the cut-off is based on the importance of timely and early detection of patients with possible swallowing deficits, which might require further specific dysphagia assessment and work-up. Items ask whether respondents experience: gagging, drooling, problems chewing, choking when you eat food, food sticking in throat or mouth, and food or liquid coming out nose or mouth. Cut-off scores above 88 mean that respondents predominately present with very few (or any) dysphagic symptoms. Conversely, a cut-off below 89 is sensitive to a dysphagia profile where respondents have (on average) experienced all of the swallowing profiles described within the SWAL-QOL. Beyond this cut-off, scores in either direction can be interpreted as suggesting an absence of dysphagia altogether $(>88)$ or increasing levels of dysphagia $(<89)$.

All CADN assessments were completed by two SLPs. SLPs were blinded to each other's CADN results and VFSS/SWAL-QOL outcomes. The CADN, VFSS and SWAL-QOL were all conducted within less than 24 hours of each other. VFSS was conducted in conjunction with a radiologist. The study received institutional approval from the Medical Ethics Board, University Hospital Tübingen, Germany (Az. 003/2015BO2). All patients, or representatives, provided written informed consent.

\section{Demonstrating methodological quality of CADN}

Criteria based on recommendations outlined in QUADAS-2 (Quality Assessment of Diagnostic Accuracy Studies - 2) [46] were used to establish the methodological quality of 
the CADN, with reference to concerns on bias and applicability. This includes the following procedural steps: (i) the reference test (VFSS for CADN part one and SWAL-QOL Symptoms for CADN part two) and the index test (CADN) were interpreted independently from each other (i.e., raters examining VFSS were blinded to outcomes of CADN); (ii) the index test was applied independently of relevant information on clinical data of the patient (assessor were not privy to information on patient severity); (iii) the reference test was applied to all patients who received the index test where practically possible; (iv) the period between the reference test and the index test was planned to be short enough (i.e., within 24 hours) to be reasonably sure that the target condition did not change between the two tests [in some cases individuals with PD may have been assessed while ON or OFF (medication / deep brain stimulation) depending on the exact time of follow-up assessment]; (v) the study population was appropriate to evaluate the proposed use of the index test; (vi) data are presented in enough detail to calculate appropriate test characteristic; (vii) the index test is described in detail so it can be reproduced; (viii) satisfactory definitions were used for normal/abnormal reference test results and normal/abnormal index test results.

\section{Statistical analysis}

Statistical analyses were conducted using SPSS using non-parametric tests. Validity, sensitivity and specificity were established separately for the two parts of CADN. Validity of the anamnesis part (CADN part one) was determined by comparing data from this part of the index tool with the "Symptoms" section of the reference test (SWAL-QOL). Validity of the consumption part (CADN part two) was determined by comparing data from this part of the index test with outcomes from the reference test (VFSS). Validity was described by analyzing sensitivity and specificity using receiver operating characteristic (ROC) graphs. Using the ROC curves, specific cut-off values for both parts of the CADN 
were used to determine test sensitivity (>.7) and specificity (>.6). We considered both specificity and sensitivity to be equally important and the compromise between those points determined the cut off for the ROC curve. Cut-offs needed to simultaneously maximize sensitivity while minimizing type II error. Sensitivity was calculated to highlight the proportion of participants who were correctly identified as presenting with clinically meaningful mealtime deficits (part one) and at risk of aspiration according to ratings derived from VFSS using PAS. Specificity calculations highlighted the proportion of participants who were correctly identified as not being at risk of aspiration. Differences between diagnostic groups and scores on the MoCA were explored. Correlations among disease severity and CADN scores were calculated using Spearman's Rho with an adjusted significance level of $\mathrm{p}<.008$ to cater for multiple comparisons.

\section{Reliability}

Inter-rater reliability for the $\mathrm{CADN}$ was established by examining agreement between rater scores on part one (anamnesis), part two (consumption) and the total CADN score from 62 participants. Differences of one point between raters were defined as an accepted deviation. Agreement was described using a Bland-Altman plot rather than intra-class correlation, as it was anticipated both samples would be strongly correlated [47]. Only data from the first assessor were included in the final validation data analysis. Repeated assessments were only used for establishing reliability, as determined in our pre-analysis plan.

\section{Item reduction}

Item reduction was not conducted for part one (anamnesis). All items on the CADN provide tangible clinical information designed to inform clinical practice. Data collected from items on dietary changes, assistance during mealtimes, frequency of coughing or choking on foods, 
liquids and saliva hold intrinsic clinical value for the management of NDD. Item reduction was only applied to the two water consumption tasks of part two as they may be eliciting the same information. Items were eliminated if they did not contribute to test accuracy.

\section{Translations}

A German translation of the English version of the CADN was developed. The translations were validated using a standard protocol that utilises both forward and backward translation with the input of multiple native English and German speakers and consensus ratings (See Supplementary Materials for English and German translations). The translation methodology has been published by other groups in different populations [48].

\section{Results}

One hundred and twenty-five patients were assessed using the CADN. 80 completed a VFSS and 94 completed the SWAL-QOL (69 completed both VFSS and SWAL-QOL). Table 1 describes patient demographic and clinical details.

Insert Table 1 about here

\section{Sensitivity and specificity}

The accuracy and utility of CADN is in part determined by how well it identifies those presenting with clinically meaningful deficits determined by clinical standards. ROC curve analysis (see methods section) shows good accuracy of .8 for DA and PD groups. The area under the curve and $95 \%$ confidence intervals CIs are summarised in Table 2 . The majority of ROC analyses were statistically significant except scores where participants received a MoCA score $<20$ or where the 'water with cup' item was removed from the total score. 
Using the ROC curve analysis, specific cut-off values for both parts of the CADN were used to determine test sensitivity and specificity. Results for sensitivity and specificity are described in detail in Table 2. Sensitivity values above .7 and specificity values above .6 were selected to ensure adequate utility of the tool as suggested by the systematic review from Kertscher et al. [22].

Sensitivity was $79.3 \%$ for PD and $86.2 \%$ for DA on part one of CADN and $85.7 \%$ for PD and 83.3\% for DA on part two of CADN. Sensitivity for all participants regardless of diagnosis was $79.3 \%$ for part one and $84.2 \%$ for part two of CADN. Specificity was $62.5 \%$ for PD and $80.0 \%$ for DA on part one of CADN and $69.8 \%$ for PD and $77.1 \%$ for DA on part two of CADN. Specificity for all participants regardless of diagnosis was $70.6 \%$ for part one and $68.9 \%$ for part two of CADN (see Table 2 for detail). Positive predictive values varied as a function of prevalence. High positive predictive values were observed for PD and DA on CADN part 1. High negative predictive values were observed for both groups for part 2. .

The area under the curve (AUC) was not significant for participants with scores less than 20 on the MoCA for part one. Removing one of the water trials from CADN part two [either task one (consumption of water via teaspoon) or task two (consumption of water via cup)] reduced the sensitivity and specificity of the tool for determining risk of aspiration according to PAS cut-offs.

Insert Table 2 about here

Inter-rater reliability 
Agreement between raters was established by assessing the same patient twice within 24 hours. Large and significant correlations were observed between raters (CADN total:

Spearman's Rho $=.674, p<.001$, part one: $R h o=.725, p<.001$, part two: $R h o=.575, p<.001)$. One-way t-test for mean CADN scores of each rater did not significantly differ (Total CADN: Mean difference=.2, $\mathrm{p}=.221$; Part 1: Mean difference=.02, $\mathrm{p}=.873$; Part 2: Mean difference $=.13, \mathrm{p}=.462$ ).

Bland-Altman plot analysis quantified the bias between mean rater assessments of the CADN and was used to estimate an agreement interval. Mean values close to zero for the total score and individual scores of part 1 and part 2 of the CADN revealed that there was no systematic offset between raters. 95\% confidence intervals of the differences between rates showed that, with rare exceptions, disagreement between raters did not go beyond \pm 2.7 for the total score, \pm 2.3 for part 1 and \pm 2.6 for part 2 . Linear regression of these measures showed there was no proportional bias (total score: $\mathrm{t}=-.646, \mathrm{p}=.52$; part one: $\mathrm{t}=-.38, \mathrm{p}=.705$; part two: $\mathrm{t}=1.0$, $\mathrm{p}=.317$ ) i.e., agreement/disagreement between raters did not depend on severity (see Figure 1).

Insert Figure 1 about here

Correlations between disease severity and dysphagia assessments

Significance was adjusted for multiple comparisons $(p<.008)$. Significant correlations were observed between CADN total, CADN part one and CADN two and measures of disease severity for PD $(\mathrm{p}<.001)$. A significant correlation was also observed between the CADN part one and disease severity ( $\mathrm{p}=.002)$ for DA (see Table 3). Correlations for the PD group 
$(R h o=.5-.67)$ were larger than the DA group which yielded small to medium correlation coefficients $(R h o=.22-.45)$.

Insert Table 3 about here

\section{Discussion}

The CADN is a reliable, valid, brief, quantifiable, and easily deployed assessment of swallowing. Here we show that the tool has good sensitivity and specificity in both PD and cerebellar neurodegenerative disorders for detecting risk of aspiration as well as clinically relevant dysphagia symptomatology. Establishment and validation of the CADN adheres to high methodological requirements of tool development [46] and components of utility and meaningfulness proposed by the FDA [49]. The CADN provides a holistic approach to dysphagia assessment by quantifying both swallowing related behaviours determined through patient interview (e.g., history of respiratory infections, assisted feeding etc.) and consumption through supervised eating and drinking of specific textures and consistencies.

To date, the majority of brief dysphagia screening assessments have sought to predict the presence or absence of aspiration without considering associated mealtime deficits [21, 22]. Aspiration risk is typically determined via trials of liquids or solids. Some use binary categories such as normal vs. abnormal (pass/fail)[13] while others deploy multiple trials of liquid in different volumes, e.g., 5-50 mL $[50,51]$. Although CADN contains water trials, it also tests consumption of puree and biscuit, thus providing a standardized assessment format to currently unstandardized clinical bedside assessments. 
The CADN demonstrated good psychometric properties, with all ROC analyses appearing statistically significant and sensitivity above $80 \%$ for PD and DA on both parts of CADN, and specificity with an average for all comparisons above 70\%. Large and significant associations were observed between the CADN with disease severity measures of PD. Smaller but meaningful effect sizes were present between CADN and disease severity measures of DA. Predictive values varied as factor of prevalence within each reference test. Higher positive predictive values were observed on CADN part 1 for both PD and DA groups where prevalence was higher (i.e., 55\% for PD and 76\% for DA). Conversely, decreased prevalence according to VFSS resulted in increased negative predictive values for CADN part 2 for PD and DA (i.e., >90). This adds to its potential value as a clinical tool for assessing dysphagia in both clinical management and clinical trials.

\section{Limitations and considerations}

Inter-rater reliability was good. It is conceivable however that reliability would have been better if repeat assessments were conducted at the same time rather than simply within 24 hours as specified given variability often observed in patients with PD whose performance can fluctuate as a function of medication routines.

Data from participants with MoCA scores <20 completing part one did not satisfy methodological requirements for adequate sensitivity or specificity. Participants with cognitive deficits tested with the MoCA did not appear to accurately answer questions relating to the impact of dysphagia. Based on these data, administration of part one of CADN is not recommended in patients with moderate to severe cognitive deficits as they may not have the capacity to properly identify aspects of their swallowing impairment. The sensitivity and specificity of part two was not overtly affected by cognition as outcomes are determined 
by the assessor, not the patient. Trial item reduction resulted in a slight decrease in sensitivity of part two when the teaspoon item was removed, however the area under the curve remained statistically significant. The test became insensitive to aspiration when the cup task was removed. Therefore, it is recommended that users administer all items of the CADN. Data show in more general terms that clinical bedside scores for dysphagia need to include tests of consumption.

Clinician derived measures of disease severity correlated significantly with all CADN scores (i.e., total, parts one and two) for patients with PD. Correlations between disease severity and the CADN were smaller for patients with DA. Only CADN part one was statistically significantly associated with changes in SARA scores. The size of correlations between CADN total, part two and disease severity measures of DA were meaningful with small to medium effect sizes (as suggested by Cohen [52]), however they were not statistically significant following adjustment for multiple comparisons. The lack of a significant association between the CADN part two suggests that some components of dysphagia are only loosely associated with overall ataxia severity. These findings differ for PD where we saw large correlations between both consumption and clinical history components of dysphagia and disease severity.

\section{Conclusions}

The dynamic and measurable nature of swallowing in NDD makes it an ideal clinical marker for monitoring disease progression and treatment response. Similarly, the requirement of the FDA to utilise patient focused outcome measures in clinical trials that reflect meaningful change in a patient's health and quality of life mean that assessments measuring swallowing constitute an important part of assessment protocols in NDD. 
Where our tool adds value over existing assessments is its capacity to identify and quantify areas of deficit requiring advice or intervention. For example, the CADN identifies the frequency of coughing/choking episodes, difficulties on particular textures/consistencies, prevalence of chest infections and whether they are related to dysphagia or standard cold and flu, whether a patient modifies their diet, or utilises alternative utensils or drinking containers to aid safe swallowing / independence. Self-reported swallowing assessments in progressive neurological disorders have capacity to provide information on dysphagia beyond the impairment level, however they can be lengthy, impractical at bedside and do not include quantification of consumption.

Given CADN is brief to administer and replicates in many aspects typical bedside assessments of swallowing, it can be adopted in a variety of clinical and research settings and multicentre trials where validated assessments are required to accurately describe the nature and severity of impairment. With additional validation it may be proven suitable for tracking disease progression or treatment response and use in NDD beyond PD and DA. Further, the CADN cut off scores help in clinical decision making processes. Scores above a certain threshold on part two are highly sensitive to risk of aspiration (scores of 5 and above on the penetration aspiration scale), suggesting the need for additional instrumental examination via FEES or VFSS.

\section{Author contributions:}

A/Prof Vogel contributed to the design of the study, collection, analysis and interpretation of the data, drafting the manuscript for intellectual content. He also supervised students, led the research team, and obtained funding for the research. 
Ms Rommel contributed to the design of the study, collected data, analysis and interpretation of the data, revising the manuscript for intellectual content, and supervision of students.

Ms Sauer contributed to the design of the study, data collection, analysis and interpretation, and revising the manuscript for intellectual content.

Dr. Horger and Dr. Krumm contributed to data collection, analysis and interpretation, and revising the manuscript for intellectual content.

Dr Himmelbach contributed to analysis and interpretation of the data and revising the manuscript for intellectual content.

Dr Synofzik contributed to the design of the study, collection, analysis and interpretation of the data, revising the manuscript for intellectual content. He also supervised students, led the research team, and obtained funding for the research.

Study funding: National Health and Medical Research Council of Australia (APV holds a Career Development Fellowship ID 1082910). The project received funding from the Alexander von Humboldt Foundation (APV), the Center for Rare Diseases (Zentrum für Seltene Erkrankungen, ZSE) Tübingen (MS and APV) and the Fondation de l' Ataxie Charlevoix-Saguenay (MS and APV).

Disclosure of all authors' financial relationships deemed relevant to the manuscript: A/Prof Vogel receives funding from the National Health and Medical Research Council, Australia (Career Development Fellowship ID 1082910), is funded by Australian Research Council grants (LP130100026, LP120200223, DP130101900), received funding from the Alexander von Humboldt Foundation and receives institutional support from The University of Melbourne.

Ms Rommel - none 
Ms Sauer - none

Dr. Horger- none

Dr. Krumm - none

Dr Himmelbach - none

Dr Synofzik received honoraria from Actelion pharmaceuticals, unrelated to the current study.

\section{References}

1. Rüb U, Brunt ER, Petrasch-Parwez E, Schöls L, Theegarten D, Auburger G, Seidel K, Schultz C, Gierga K, Paulson H, Van Broeckhoven C, Deller T and De Vos RAI. Degeneration of ingestion-related brainstem nuclei in spinocerebellar ataxia type 2, 3, 6 and 7. Neuropathology and Applied Neurobiology 2006: 32:635-49. doi 10.1111/j.1365-2990.2006.00772.x

2. Altman KW, Richards A, Goldberg L, Frucht S and McCabe DJ. Dysphagia in Stroke, Neurodegenerative Disease, and Advanced Dementia. Otolaryngologic Clinics of North America 2013: 46:1137-49. doi http://dx.doi.org/10.1016/j.otc.2013.08.005

3. Ekberg O, Hamdy S, Woisard V, Wuttge-Hannig A and Ortega P. Social and Psychological Burden of Dysphagia: Its Impact on Diagnosis and Treatment. Dysphagia 2002: 17:139-46. doi 10.1007/s00455-001-0113-5

4. Hely MA, Reid WG, Adena MA, Halliday GM and Morris JG. The Sydney multicenter study of Parkinson's disease: The inevitability of dementia at 20 years. Movement Disorders 2008: 23:837-44. doi 10.1002/mds.21956

5. Heemskerk A-W and Roos RA. Aspiration pneumonia and death in Huntington's disease. PLoS Currents 2012: 4:RRN1293. doi 10.1371/currents.RRN1293

6. Vogel AP, Pointon L, Maule R and Churchyard AJ. Clinical features of dysphagia and swallowing deficits in Huntington's Disease: A descriptive videofluoroscopic study. Clinical Genetics 2011: 80:60.

7. Higo R, Tayama $\mathrm{N}$ and Nito $\mathrm{T}$. Longitudinal analysis of progression of dysphagia in amyotrophic lateral sclerosis. Auris Nasus Larynx 2004: 31:247-54. doi http://dx.doi.org/10.1016/j.anl.2004.05.009

8. Tsou AY, Paulsen EK, Lagedrost SJ, Perlman SL, Mathews KD, Wilmot GR, Ravina B, Koeppen AH and Lynch DR. Mortality in Friedreich Ataxia. Journal of the Neurological Sciences 2011: 307:46-9. doi http://dx.doi.org/10.1016/j.jns.2011.05.023

9. Vogel AP, Fendel L, Brubacher KP, Chan V and Maule R. Dysphagia in spinocerebellar ataxia and multiple system atrophy-cerebellar. Speech, Language and Hearing 2015: 18:39-43. doi doi:10.1179/2050572814Y.0000000047

10. Vogel AP, Brown SE, Folker JE, Corben LA and Delatycki MB. Dysphagia and swallowing related quality of life in Friedreich ataxia. J Neurol 2014: 261:392-9. 
11. Mathers-Schmidt BA and Kurlinski M. Dysphagia Evaluation Practices: Inconsistencies in Clinical Assessment and Instrumental Examination DecisionMaking. Dysphagia: 18:114-25. doi 10.1007/s00455-002-0094-Z

12. Clavé P, Arreola V, Romea M, Medina L, Palomera E and Serra-Prat M. Accuracy of the volume-viscosity swallow test for clinical screening of oropharyngeal dysphagia and aspiration. Clinical Nutrition 2008: 27:806-15. doi http://dx.doi.org/10.1016/j.clnu.2008.06.011

13. Martino R, Silver F, Teasell R, Bayley M, Nicholson GA, Streiner DL and Diamant NE. The Toronto Bedside Swallowing Screening Test (TOR-BSST): Development and Validation of a Dysphagia Screening Tool for Patients With Stroke. Stroke 2009: 40:555-61. doi 10.1161/strokeaha.107.510370

14. Paris G, Martinaud O, Hannequin D, Petit A, Cuvelier A, Guedon E, Ropenneck P and Verin E. Clinical screening of oropharyngeal dysphagia in patients with ALS. Annals of Physical and Rehabilitation Medicine 2012: 55:601-8. doi http://dx.doi.org/10.1016/j.rehab.2012.10.005

15. Suiter DM and Leder SB. Clinical Utility of the 3-ounce Water Swallow Test. Dysphagia 2008: 23:244-50. doi 10.1007/s00455-007-9127-y

16. Wakasugi Y, Tohara H, Hattori F, Motohashi Y, Nakane A, Goto S, Ouchi Y, Mikushi S, Takeuchi S and Uematsu H. Screening Test for Silent Aspiration at the Bedside. Dysphagia 2008: 23:364-70. doi 10.1007/s00455-008-9150-7

17. Kagaya H, Okada S, Saitoh E, Baba M, Yokoyama M and Takahashi H. Simple Swallowing Provocation Test Has Limited Applicability as a Screening Tool for Detecting Aspiration, Silent Aspiration, or Penetration. Dysphagia 2010: 25:6-10. doi 10.1007/s00455-009-9222-3

18. Cohen JT and Manor Y. Swallowing disturbance questionnaire for detecting dysphagia. The Laryngoscope 2011: 121:1383-7. doi 10.1002/lary.21839

19. Trapl M, Enderle P, Nowotny M, Teuschl Y, Matz K, Dachenhausen A and Brainin M. Dysphagia Bedside Screening for Acute-Stroke Patients: The Gugging Swallowing Screen. Stroke 2007: 38:2948-52. doi 10.1161/strokeaha.107.483933

20. Mann GD. MASA: the Mann Assessment of Swallowing Ability. In: G. D. Mann, editor. Dysphagia Series. New York, NY, Singular Thomson Learning; 2002. pp. 56.

21. Bours GJ, Speyer R, Lemmens J, Limburg M and De Wit R. Bedside screening tests vs. videofluoroscopy or fibreoptic endoscopic evaluation of swallowing to detect dysphagia in patients with neurological disorders: systematic review. Journal of Advanced Nursing 2009: 65:477-93. doi 10.1111/j.1365-2648.2008.04915.x

22. Kertscher B, Speyer R, Palmieri M and Plant C. Bedside Screening to Detect Oropharyngeal Dysphagia in Patients with Neurological Disorders: An Updated Systematic Review. Dysphagia 2014: 29:204-12. doi 10.1007/s00455-013-9490-9

23. Logemann JA, Veis S and Colangelo L. A Screening Procedure for Oropharyngeal Dysphagia. Dysphagia 1999: 14:44-51. doi 10.1007/PL00009583

24. Daniels SK, McAdam CP, Brailey K and Foundas AL. Clinical Assessment of Swallowing and Prediction of Dysphagia Severity. American Journal of SpeechLanguage Pathology 1997: 6:17-24. doi 10.1044/1058-0360.0604.17

25. Leder SB and Espinosa JF. Aspiration Risk After Acute Stroke: Comparison of Clinical Examination and Fiberoptic Endoscopic Evaluation of Swallowing. Dysphagia 2002: 17:214-8. doi 10.1007/s00455-002-0054-7

26. McCullough GH, Wertz RT and Rosenbek JC. Sensitivity and specificity of clinical/bedside examination signs for detecting aspiration in adults subsequent to stroke. Journal of Communication Disorders 2001: 34:55-72. doi http://dx.doi.org/10.1016/S0021-9924(00)00041-1 
27. Keage MJ, Delatycki M, Corben LA and Vogel AP. A Systematic Review of Selfreported Swallowing Assessments in Progressive Neurological Disorders. Dysphagia 2015: 30:27-46. doi 10.1007/s00455-014-9579-9

28. Enderby P and Palmer R. Frenchay Dysarthria Assessment - Second Edition. Austin, TX, Pro-Ed; 2008.

29. Synofzik M, Soehn AS, Gburek-Augustat J, Schicks J, Karle KN, Schüle R, Haack TB, Schöning M, Biskup S, Rudnik-Schöneborn S, Senderek J, Hoffmann KT, MacLeod P, Schwarz J, Bender B, Krüger S, Kreuz F, Bauer P and Schöls L. Autosomal recessive spastic ataxia of Charlevoix Saguenay (ARSACS): expanding the genetic, clinical and imaging spectrum. Orphanet journal of rare diseases 2013: $8: 41$.

30. Synofzik M, Srulijes K, Godau J, Berg D and Schöls L. Characterizing POLG Ataxia: Clinics, Electrophysiology and Imaging. Cerebellum 2012: 11:1002-11. doi 10.1007/s12311-012-0378-2

31. Goetz CG, Poewe W, Rascol O, Sampaio C, Stebbins GT, Counsell C, Giladi N, Holloway RG, Moore CG, Wenning GK, Yahr MD and Seidl L. Movement Disorder Society Task Force report on the Hoehn and Yahr staging scale: Status and recommendations The Movement Disorder Society Task Force on rating scales for Parkinson's disease. Movement Disorders 2004: 19:1020-8. doi 10.1002/mds.20213

32. Schmitz-Hubsch T, Du Montcel ST, Baliko L, Berciano J, Boesch S, Depondt C, Giunti P, Globas C, Infante J, Kang J-S, Kremer B, Mariotti C, Melegh B, Pandolfo M, Rakowicz M, Ribai P, Rola R, Schols L, Szymanski S, Van De Warrenburg BP, Durr A and Klockgether T. Scale for the assessment and rating of ataxia: Development of a new clinical scale. Neurology 2006: 66:1717-20.

33. Katz S. Assessing Self-maintenance: Activities of Daily Living, Mobility, and Instrumental Activities of Daily Living. Journal of the American Geriatrics Society 1983: 31:721-7. doi 10.1111/j.1532-5415.1983.tb03391.x

34. Nasreddine ZS, Phillips NA, Bédirian V, Charbonneau S, Whitehead V, Collin I, Cummings JL and Chertkow H. The Montreal Cognitive Assessment, MoCA: A Brief Screening Tool For Mild Cognitive Impairment. Journal of the American Geriatrics Society 2005: 53:695-9. doi 10.1111/j.1532-5415.2005.53221.x

35. McHorney CA, Martin-Harris B, Robbins JA and Rosenbek JC. Clinical Validity of the SWAL-QOL and SWAL-CARE Outcome Tools with Respect to Bolus Flow Measures. Dysphagia 2006: 21:141-8. doi 10.1007/s00455-005-0026-9

36. Rosenbek JC, Robbins JA, Roecker EB, Coyle JL and Wood JL. A penetrationaspiration scale. Dysphagia 1996: 11:93-8. doi 10.1007/bf00417897

37. McHorney CA, Bricker DE, Kramer AE, Rosenbek JC, Robbins J, Chignell KA, Logemann JA and Clarke C. The SWAL-QOL Outcomes Tool for Oropharyngeal Dysphagia in Adults: I. Conceptual Foundation and Item Development. Dysphagia 2000: 15:115-21. doi 10.1007/s004550010012

38. McHorney CA, Bricker DE, Robbins J, Kramer AE, Rosenbek JC and Chignell KA. The SWAL-QOL Outcomes Tool for Oropharyngeal Dysphagiain Adults: II. Item Reduction and Preliminary Scaling. Dysphagia 2000: 15:122-33. doi $10.1007 / \mathrm{s} 004550010013$

39. McHorney CA, Robbins JA, Lomax K, Rosenbek JC, Chignell K, Kramer AE and Earl Bricker D. The SWAL-QOL and SWAL-CARE Outcomes Tool for Oropharyngeal Dysphagia in Adults: III. Documentation of Reliability and Validity. Dysphagia 2002: 17:97-114. doi 10.1007/s00455-001-0109-1

40. Leow LP, Huckabee M-L, Anderson T and Beckert L. The Impact of Dysphagia on Quality of Life in Ageing and Parkinson's Disease as Measured by the Swallowing 
Quality of Life (SWAL-QOL) Questionnaire. Dysphagia 2010: 25:216-20. doi 10.1007/s00455-009-9245-9

41. Plowman-Prine EK, Sapienza CM, Okun MS, Pollock SL, Jacobson C, Wu SS and Rosenbek JC. The relationship between quality of life and swallowing in Parkinson's disease. Movement Disorders 2009: 24:1352-8. doi 10.1002/mds.22617

42. Russo AD, Reckziegel ER, Krum-Santos AC, Augustin MC, Scheeren B, Freitas CD, Torman VL, Saraiva-Pereira M-L, Saute JA and Jardim LB. Clinical Scales Predict Significant Videofluoroscopic Dysphagia in Machado Joseph Disease Patients. Movement Disorders Clinical Practice 2015:n/a-n/a. doi 10.1002/mdc3.12173

43. Paris G, Martinaud O, Petit A, Cuvelier A, Hannequin D, Roppeneck P and Verin E. Oropharyngeal dysphagia in amyotrophic lateral sclerosis alters quality of life. Journal of Oral Rehabilitation 2013: 40:199-204. doi 10.1111/joor.12019

44. Reyes A, Cruickshank T, Nosaka K and Ziman M. Respiratory muscle training on pulmonary and swallowing function in patients with Huntington's disease: a pilot randomised controlled trial. Clinical Rehabilitation 2015: 29:961-73. doi doi:10.1177/0269215514564087

45. Vogel AP, Rommel N, Oettinger A, Horger M, Krumm P, Kraus E-M, Schöls L and Synofzik M. Speech and swallowing abnormalities in adults with POLG associated ataxia (POLG-A). Mitochondrion 2017.

46. Whiting PF, Rutjes AW, Westwood ME, Mallett S, Deeks JJ, Reitsma JB, Leeflang MM, Sterne JA and Bossuyt PM. QUADAS-2: A Revised Tool for the Quality Assessment of Diagnostic Accuracy Studies. Annals of Internal Medicine 2011: 155:529-36. doi 10.7326/0003-4819-155-8-201110180-00009

47. Giavarina D. Understanding Bland Altman analysis. Biochemia Medica 2015: 25:141-51. doi 10.11613/BM.2015.015

48. CochKrismer F, Duerr S, Minnerop M, Klockgether T, Stamelou M, Eggert KM, Oertel WH, Schrag A, Poewe W and Wenning GK. [MSA-QoL: disease-specific questionnaire to assess health-related quality of life in multiple system atrophy: validation of the German translation]. Der Nervenarzt 2013: 84:709-14.

49. FDA. Guidance for Industry Patient-Reported Outcome Measures: Use in Medical Product Development to Support Labeling Claims. In: U. F. a. D. Administration, editor. Washington DC, Food and Drug Administration; 2009.

50. Lim SH, Lieu PK, Phua SY, Seshadri R, Venketasubramanian N, Lee SH and Choo PW. Accuracy of Bedside Clinical Methods Compared with Fiberoptic Endoscopic Examination of Swallowing (FEES) in Determining the Risk of Aspiration in Acute Stroke Patients. Dysphagia 2001: 16:1-6. doi 10.1007/s004550000038

51. Chong MS, Lieu PK, Sitoh YY, Meng YY and Leow LP. Bedside clinical methods useful as screening test for aspiration in elderly patients with recent and previous strokes. Ann Acad Med Singapore 2003: 32:790-4.

52. Cohen J. Statistical Power Analysis for the Behavioral Sciences. Hillsdale, NJ: Lawrence Erlbaum; 1988. 
Table 1: Patient demographic and clinical information (Mean \pm Standard Deviation (range))

\begin{tabular}{l|lll}
\hline Variable & PD & DA & All \\
\hline$N$ & 60 & 65 & 125 \\
\hline Sex (m,f) & 40,20 & 35,30 & 75,50 \\
\hline Age (y) & $68.8 \pm 10.1(39-86)$ & $47.4 \pm 15.3(18-85)$ & $57.7 \pm 19.9$ \\
\hline Disease severity & $2.8 \pm .8(1-5)(\mathrm{HY})$ & $\begin{array}{l}16.2 \pm 7.7(1-32) \\
(\text { SARA })\end{array}$ & na \\
\hline Disease duration $(\mathrm{y})$ & $10.9 \pm 7.8(0-36)$ & $12.7 \pm 7.9(1-27)$ & $11.7 \pm 7.9$ \\
\hline MoCA $^{\text {IADL }}{ }^{\mathrm{a}}$ & $23.6 \pm 5.8(6-30)$ & $26.3 \pm 3.8(13-30)$ & $25.2 \pm 4.9$ \\
\hline PAS $^{\mathrm{b}}$ & $4.9 \pm 1.6(0-6)$ & $3.1 \pm 2.4(0-6)$ & $4.8 \pm 1.6$ \\
\hline SWAL-QOL & $2.7 \pm 2.3(1-8)^{\mathrm{c}}$ & $3.1 \pm 2.4(1-8)^{\mathrm{d}}$ & $3.0 \pm 2.3$ \\
\hline Completed VFSS and & $85.2 \pm 13.2(57-100)^{\mathrm{e}}$ & $82.1 \pm 13.0(40-100)^{\mathrm{f}}$ & $83.8 \pm 13.2$ \\
\hline SWAL-QOL $(\%)$ & 55,88 & 74,63 & 64,75 \\
\hline a & & & \\
\hline
\end{tabular}

${ }^{\mathrm{a}}$ Higher scores indicate more independence; ${ }^{\mathrm{b}}$ Lower scores indicate better performance; ${ }^{\mathrm{c}}$ $21 \%$ of PD patients with PAS values $\geq 5 ;{ }^{\mathrm{d}} 25 \%$ of DA patients with PAS values $\geq 5$; ${ }^{\mathrm{e}} 55 \%$ of PD patients with SWAL-QOL values $<89 ;{ }^{\mathrm{f}} 76 \%$ of DA patients with SWAL-QOL values $<89$; na= not available as measured by different tools; $\mathrm{PD}=$ Parkinson's Disease; DA, degenerative ataxia; MoCA = Montreal Cognitive Assessment, IADL= instrumental activities of daily living, PAS= Penetration Aspiration Scale, VFSS= videofluoroscopic swallowing study, HY= Hoehn and Yahr Scale, SARA= Scale for the Assessment and Rating of Ataxia.

Table 2: Area under the curve, sensitivity, specificity and predictive values of CADN parts one and two

\begin{tabular}{l|llllll}
\hline $\begin{array}{l}\text { Disease } \\
\text { group }\end{array}$ & $\begin{array}{l}\text { CADN } \\
\text { part }\end{array}$ & $\begin{array}{l}\text { CADN } \\
\text { Cut-points }\end{array}$ & $\begin{array}{l}\text { Sensitivity } \\
(\%)\end{array}$ & $\begin{array}{l}\text { Specificity } \\
(\%)\end{array}$ & $\begin{array}{l}\text { Area under the curve (CI } \\
95 \%)\end{array}$ & $\begin{array}{l}\text { Positive pred } \\
\text { values (CI 95 }\end{array}$ \\
\hline All & $1^{*}$ & $\geq 1.75$ & 79.3 & 70.6 & $.81(.72-.9) \mathrm{p}<.001$ & $82.14(72.89-$ \\
\hline & $2^{\#}$ & $\geq 1.5$ & 84.2 & 68.9 & $.79(.67-.91) \mathrm{p}<.001$ & $45.71(35.6-5$ \\
\hline PD & $1^{*}$ & $\geq 1.25$ & 79.3 & 62.5 & $.8(.69-.92) \mathrm{p}<.001$ & $71.87(59.61-$ \\
\hline & $2^{\#}$ & $\geq 1.5$ & 85.7 & 69.8 & $.8(.59-1.00) \mathrm{p}=.016$ & $42.86(28.11-$ \\
\hline DA & $1^{*}$ & $\geq 1.75$ & 86.2 & 80.0 & $.8(.61-.99) \mathrm{p}=.005$ & $91.31(77.46-$ \\
\hline & $2^{\#}$ & $\geq 2.5$ & 83.3 & 77.1 & $.79(.64-.94) \mathrm{p}=.003$ & $55.56(39.27-$ \\
\hline MoCA $\geq 20$ & $\begin{array}{l}1^{*} \\
(\mathrm{n}=82)\end{array}$ & $\geq 1.75$ & 81.6 & 71.0 & $.83(.74-.92) \mathrm{p}<.001$ & - \\
\hline & $\begin{array}{l}2^{\#} \\
(\mathrm{n}=70)\end{array}$ & $\geq 1.5$ & 81.3 & 70.4 & $.77(.64-.91) \mathrm{p}=.001$ & - \\
\hline $\begin{array}{l}\text { MoCA }<20 \\
1^{*} \\
(\mathrm{n}=12)\end{array}$ & $\geq 1.0$ & 66.7 & 66.7 & $.74(.45-1.00), \mathrm{p}=.23$ & - \\
\hline $\begin{array}{l}\text { All not } \\
\text { incl. item } \\
\text { water with }\end{array}$ & $\begin{array}{l}2^{\#} \\
(\mathrm{n}=10)\end{array}$ & $\geq 3.5$ & 100.0 & 85.7 & $.95(.82-1.00), \mathrm{p}=.03$ & - \\
\hline
\end{tabular}




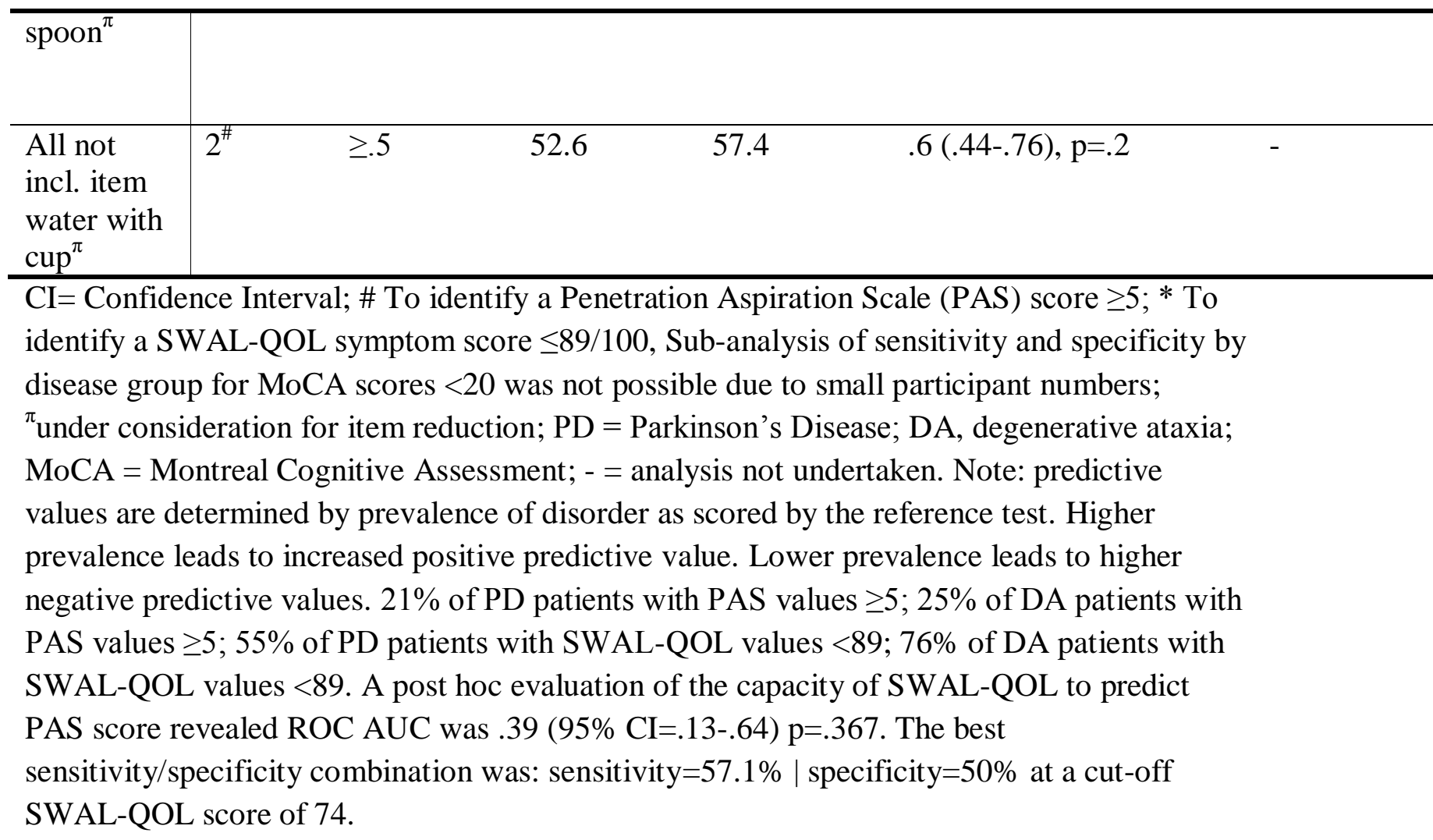

Table 3: Correlation with disease severity: Spearman's Rho (95\% confidence interval)

\begin{tabular}{lll}
\hline & PD $(H Y)$ & DA (SARA) \\
\hline CADN Total & $\mathbf{. 6 6 7}(.5-.79) \mathbf{p}<.001$ & $.331(.09-.55) p=.028$ \\
CADN Part 1 & $\mathbf{. 5}(.28-.7) \mathbf{p}=\mathbf{. 0 0 2}$ & $\mathbf{. 4 5}(.27-.6) \mathbf{p}=\mathbf{. 0 0 2}$ \\
CADN Part 2 & $\mathbf{. 6 3 7}(. \mathbf{4 6 - . 7 7 )} \mathbf{p}<. \mathbf{0 0 1}$ & $.221(.01-.44) \mathrm{p}=.149$ \\
\hline
\end{tabular}

$\mathrm{PD}=$ Parkinson's disease, DA, degenerative ataxia, $\mathrm{HY}=$ Hoehn and Yahr Scale, SARA= Scale for the Assessment and Rating of Ataxia, bold values indicate correlations reaching significance after adjustment for multiple comparisons $(\mathrm{p}<.008)$

Figure 1: Bland-Altman plots of rater agreement

Mean values close to zero for the total score and individual scores of part 1 and part 2 of the CADN revealed that there was no systematic offset between raters. $95 \%$ confidence intervals of the differences between rates showed that, with rare exceptions, disagreement between raters did not go beyond \pm 2.7 for the total score, \pm 2.3 for part 1 and \pm 2.6 for part 2 . 

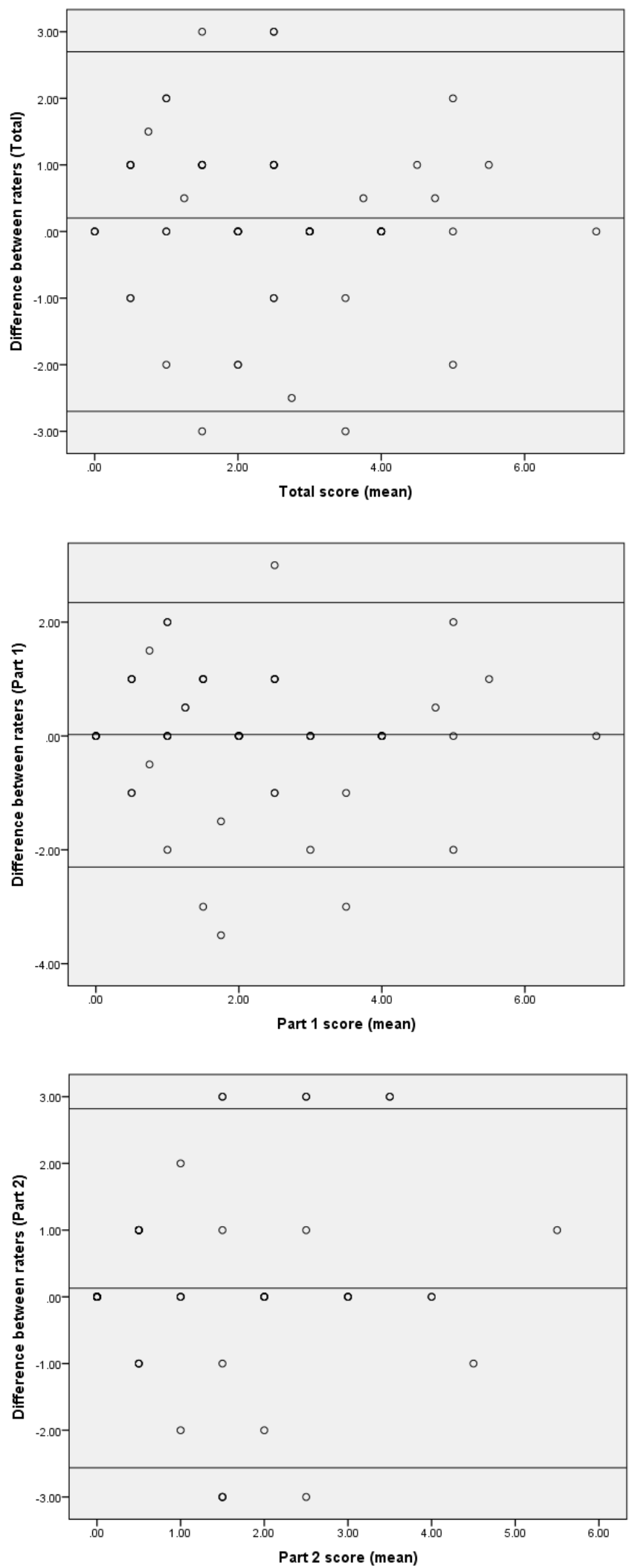
